\title{
Heavy-light mesons in 2+1 flavor lattice QCD
}

\section{Y. Namekawa* for PACS-CS Collaboration,}

Center for Computational Sciences, University of Tsukuba, Tsukuba, Ibaraki 305-8577, Japan

Heavy-light meson system is investigated using the relativistic heavy quark action on the $2+1$ dynamical flavor PACS-CS configurations at the lattice spacing $a^{-1}=2.2 \mathrm{GeV}$ and the spatial extent $L=3 \mathrm{fm}$. Dynamical up-down and strange quark masses as well as the valence charm quark mass are set around their physical values. We measure the charm- $u d$ and charm-strange meson masses and decay constants. Our results are consistent with the experimental values except the hyperfine splitting of the charm-strange meson. We also estimate the CKM matrix elements in the second row.

The XXVII International Symposium on Lattice Field Theory - LAT2009

July 26-31 2009

Peking University, Beijing, China

\footnotetext{
* Speaker.

${ }^{\dagger}$ E-mail: namekawa@ccs.tsukuba.ac.jp
} 


\section{Introduction}

Precise determination of the CKM matrix is an important step to establish the validity range of the Standard Model, thereby setting the basis for exploring physics at smaller space-time scales. In this respect the CKM matrix elements in the second row, especially $\left|V_{c d}\right|$ and $\left|V_{c s}\right|$, are still poorly determined with errors in the $10 \%$ range [1]. This situation contrasts sharply with those of the first row where $\left|V_{u d}\right|$ and $\left|V_{u s}\right|$ are known at sub percent level.

Lattice QCD provides a variety of means for precise determination of the second row of the CKM matrix. With $\left|V_{c s}\right|$, for example, the leptonic decay width of the $D_{s}$ meson $\Gamma\left(D_{s} \rightarrow l v\right)$ is given by

$$
\Gamma\left(D_{s} \rightarrow l v\right)=\frac{G_{F}^{2}}{8 \pi} m_{l}^{2} m_{D_{s}} f_{D_{s}}^{2}\left(1-\frac{m_{l}^{2}}{m_{D_{s}}^{2}}\right)^{2}\left|V_{c s}\right|^{2} .
$$

so that a lattice determination of the decay constant $f_{D_{s}}$ combined with the experimental value of $\Gamma\left(D_{s} \rightarrow l v\right)$ will allow us to extract $\left|V_{c s}\right|$. The other matrix element $\left|V_{c d}\right|$ can be obtained in the same way.

An intriguing topic in the charm quark sector is a disagreement of the value of the $D_{s}$ meson decay constant from lattice QCD and experiment. In 2007 HPQCD and UKQCD Collaboration reported a calculation of this quantity using the HISQ action for heavy quark on the $2+1$ dynamical flavor MILC configurations [2]. Their value $f_{D_{s}}=241 \pm 3 \mathrm{MeV}$ exhibited significant deviation from those of the CLEO and Belle experiments at the time. The latest CLEO value [3] $f_{D_{s}}=$ $259.5 \pm 7.3 \mathrm{MeV}$, though smaller than 2007 , is still two standard deviations away.

FNAL Collaboration also calculated the decay constant on the MILC configurations using the clover heavy quark formalism. Their original value $f_{D_{s}}=249(3)(16) \mathrm{MeV}$ [4] was also smaller than experiment, but an update this year [5] including a $2 \%$ change in the scale setting of the MILC ensemble moved the value up to $f_{D_{s}}=260(10) \mathrm{MeV}$.

In this paper we present a status report of our work on the charmed mesons using the relativistic heavy quark formalism on the $2+1$ dynamical flavor PACS-CS configurations.

\section{Set up}

The charm quark system is simulated with a relativistic heavy quark action on the $2+1$ flavor lattice QCD configurations. The configurations are generated by the PACS-CS Collaboration [6] using the nonperturbatively $O(a)$-improved Wilson quark action with $c_{\mathrm{SW}}^{\mathrm{NP}}=1.715$ [7] and the Iwasaki gauge action. The lattice size is $32^{3} \times 64$ whose spatial extent is $L=3 \mathrm{fm}$ with the lattice spacing of $a=0.09 \mathrm{fm}$. The dynamical up-down quark masses range from $8 \mathrm{MeV}$ down to 3 $\mathrm{MeV}$, which is close to the the physical value. We utilize the same quark action for the light quark propagators in our calculation.

For the heavy quarks, we employ a relativistic heavy quark action proposed in Ref. [8]. The cutoff errors are reduced from $O\left(\left(m_{Q} a\right)^{n}\right)$ to $O\left(f\left(m_{Q} a\right)\left(a \Lambda_{Q C D}\right)^{2}\right)$ where $f\left(m_{Q} a\right)$ is an analytic function around $m_{Q} a=0$. We use one-loop perturbative values for parameters in the heavy quark action [9]. In addition, nonperturbative contributions at the massless limit is included for the clover 
Table 1: Simulation parameters. Quark masses are perturbatively renormalized in the $\overline{\mathrm{MS}}$ scheme. The renormalization scale is $\mu=1 / a$ for each simulation point and $\mu=2 \mathrm{GeV}$ for the physical point.

\begin{tabular}{cccccc}
\hline$\kappa_{\mathrm{ud}}$ & $\kappa_{\mathrm{s}}$ & $m_{\mathrm{ud}}^{\overline{\mathrm{MS}}}(\mu)[\mathrm{MeV}]$ & $m_{\mathrm{s}}^{\overline{\mathrm{MS}}}(\mu)[\mathrm{MeV}]$ & \multicolumn{2}{c}{ \#conf } \\
& & & & measured & MD time/total \\
\hline 0.13781 & 0.13640 & $3.5(2)$ & $86.4(1)$ & 65 & $1625 / 1625$ \\
0.13770 & 0.13660 & $8.3(5)$ & $74.1(4)$ & 60 & $1500 / 1500$ \\
0.137785 & 0.13660 & $3.5(1)$ & $72.8(2)$ & 200 & $1000 / 2000$ \\
\hline \multicolumn{2}{c}{ physical point } & $2.53(5)$ & $72.7(8)$ & & \\
\hline
\end{tabular}
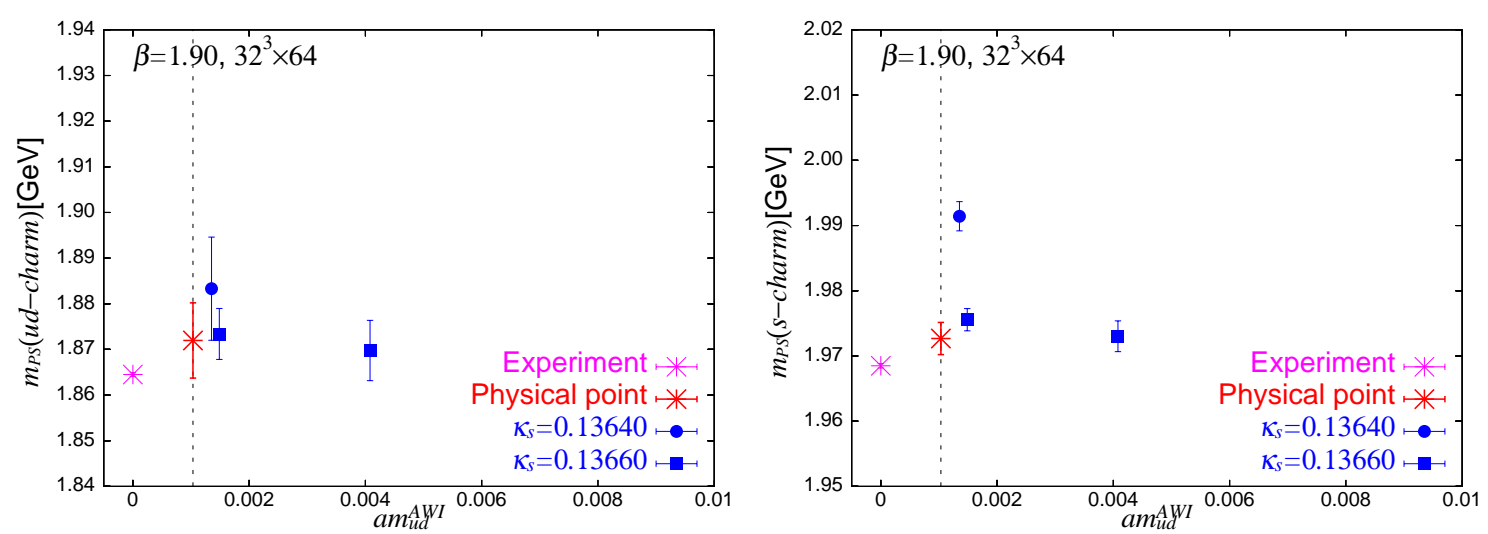

Figure 1: $m_{\mathrm{ud}}^{\text {sea }}$ quark mass dependence of $m_{D}$ (left panel) and $m_{D_{s}}$ (right panel).

coefficients, and the parameter $v$ is adjusted nonperturbatively to reproduce the relativistic dispersion relation of the charmonium. Our valence heavy quark masses are always tuned to the physical charm quark mass point, which is determined by the spin-averaged $1 S$ state of the charmonium, $M(1 S)=\left(M_{\eta_{c}}+3 M_{J / \psi}\right) / 4=3.0677(3) \mathrm{GeV}$. Our results for charmonium spectrum have been reported in Ref. [10].

Table 1 summarizes our simulation parameters and the statistics of the configuration sets we have used for the heavy quark measurements. The number of the source points is quadrupled to suppress statistical fluctuations.

\section{Results for $D$ and $D_{s}$ meson masses and decay constants}

We present our results for $D$ and $D_{s}$ meson masses and their decay constants. Since the physical charm quark mass has already been fixed with the heavy-heavy spectrum, all heavy-light meson quantities can be predicted.

Figure 1 compares our results for the $D$ and $D_{s}$ meson masses with the experimental values [3]. Since our sea quark masses are close to the physical values, we extrapolate our results with a linear function of the up-down and the strange quark masses to the physical point,

$$
M=A+B\left(m_{\mathrm{ud}}-m_{\mathrm{ud}}^{\mathrm{phys}}\right)+C\left(m_{\mathrm{s}}-m_{\mathrm{s}}^{\text {phys }}\right) .
$$



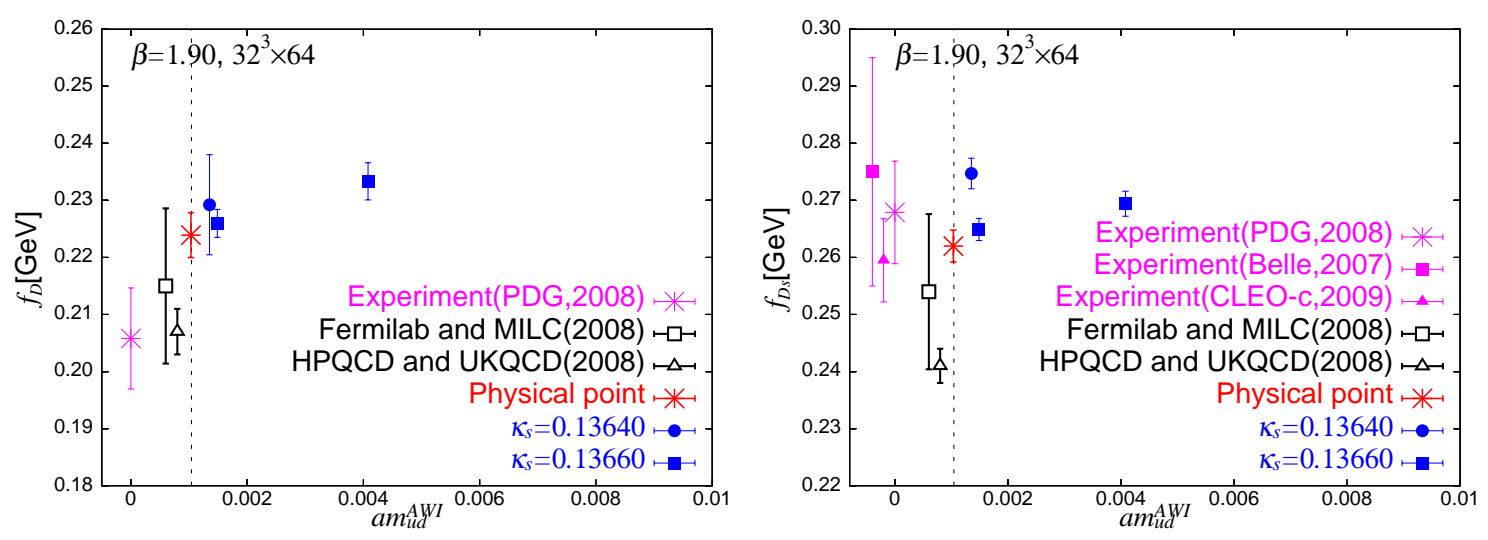

Figure 2: $m_{\mathrm{ud}}^{\text {sea }}$ quark mass dependence of $f_{D}\left(\right.$ left panel) and $f_{D_{s}}$ (right panel).

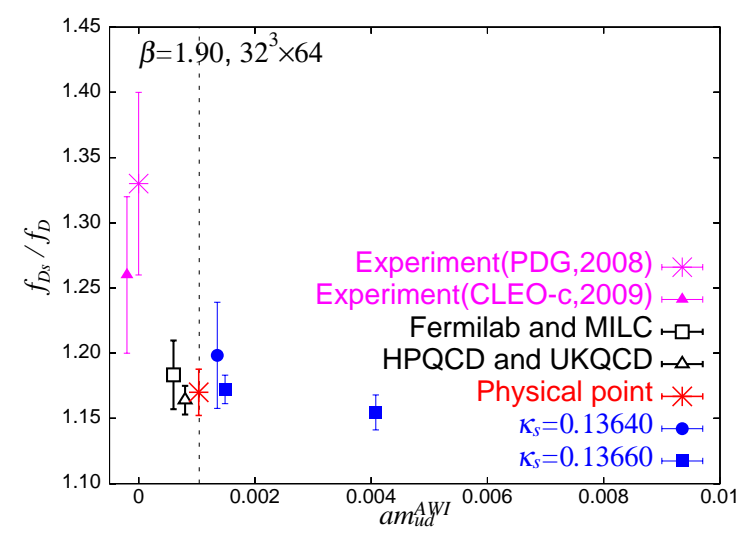

Figure 3: $m_{\mathrm{ud}}^{\text {sea }}$ quark mass dependence of $f_{D_{s}}$ over $f_{D}$.

Our results at the physical point (red bursts) reproduce the experimental spectrum well.

We also calculate the decay constants of heavy-light pseudoscalar mesons,

$$
\begin{aligned}
<0\left|A_{\mu}^{i m p}\right| P S(p)>= & i f_{P S} p_{\mu}, \\
A_{\mu}^{i m p}= & \sqrt{2 \kappa_{q}} \sqrt{2 \kappa_{Q}} Z_{A_{\mu}}\left\{\bar{q}(x) \gamma_{\mu} \gamma_{5} Q(x)\right. \\
& \left.-c_{A_{\mu}}^{+} \partial_{\mu}^{+}\left(\bar{q}(x) \gamma_{\mu} \gamma_{5} Q(x)\right)-c_{A_{\mu}}^{-} \partial_{\mu}^{-}\left(\bar{q}(x) \gamma_{\mu} \gamma_{5} Q(x)\right)\right\} .
\end{aligned}
$$

For the renormalization factor and the improvement coefficients of the axial current, we employ one-loop perturbative values [11]. Furthermore, the nonperturbative contribution at the massless limit is incorporated to the improvement coefficient $c_{A_{4}}^{+}$by writing

$$
c_{A_{4}}^{+}=\left(c_{A_{4}}^{+}\left(m_{Q} a\right)-c_{A_{4}}^{+}(0)\right)^{\mathrm{PT}}+c_{A}^{\mathrm{NP}}
$$

with $c_{A}^{\mathrm{NP}}=-0.03876106[12]$.

Figure 2 shows the decay constants, where we also plot other $2+1$ flavor lattice QCD results from HPQCD and UKQCD Collaboration [2] and FNAL group [5], as well as the experimental values $[1,3,13]$, for comparison. Our value for $f_{D_{s}}$ agrees with the experimental determinations, while 

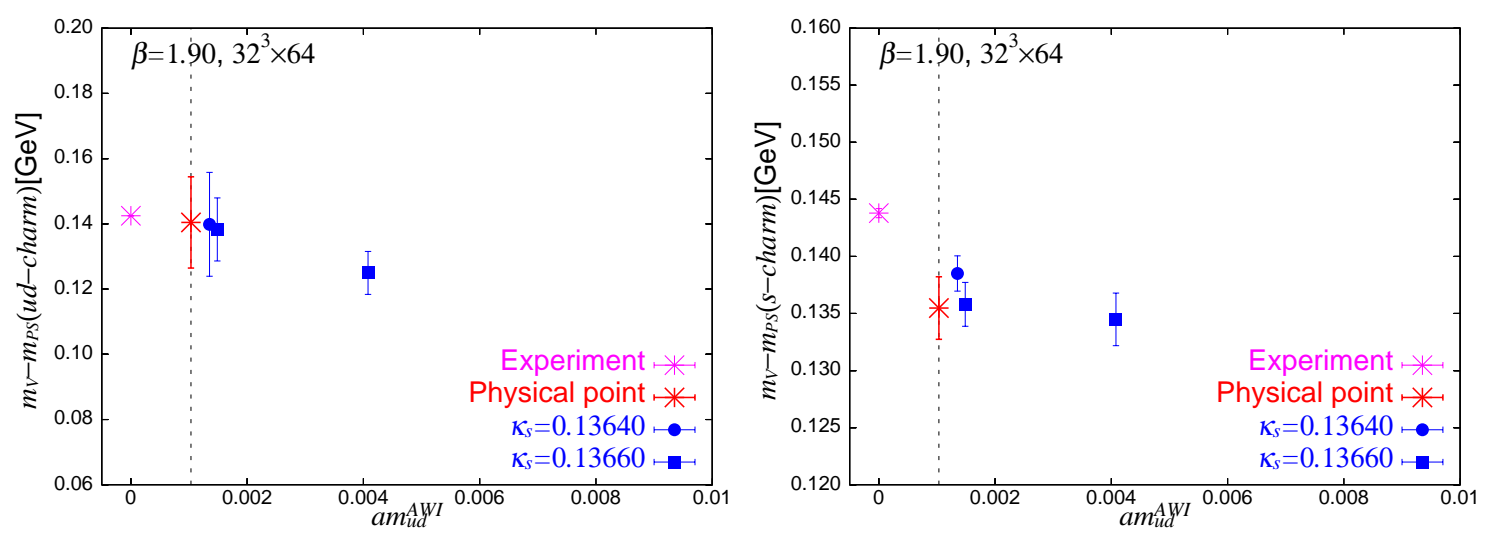

Figure 4: $m_{\mathrm{ud}}^{\text {sea }}$ quark mass dependence of hyperfine splittings for charm- $u d$ (left panel) and and charmstrange mesons (right panel).

that for $f_{D}$ is somewhat larger. Comparing the three sets of lattice determinations, we observe, both for $f_{D}$ and $f_{D_{s}}$, an agreement between our values and those of the FNAL group, while there seems to be a clear discrepancy between our values and that by the HPQCD and UKQCD Collaboration. Possible reasons are our use of perturbative renormalization factors and the necessity of taking the continuum extrapolation.

In Fig. 3 we plot the ratios of $f_{D_{s}}$ to $f_{D}$ in which uncertainties coming from the perturbative renormalization factors cancel out, and perhaps that of the lattice cutoff to some extent. It is interesting that all three lattice results are mutually consistent with small errors of a few percent, but that they seem to lie at the lower edge of the experimental value.

\section{Results of hyperfine splittings for charm- $u d$ and charm-strange mesons}

We evaluate the hyperfine splittings for charm- $u d$ and charm-strange mesons. Masses of $D^{*}$ and $D_{s}^{*}$ are obtained from their two-point functions. $D^{*}$ and $D_{s}^{*}$ decays are prohibited energetically on our lattice.

Figure 4 represents our results for the hyperfine splittings with the experimental values [1]. For $D^{*}-D$ mass difference, our result at the physical point (red bursts) reproduces the experimental spectrum. On the other hand, $D_{s}^{*}-D_{s}$ mass difference shows $10 \%$ deviation from the experimental value. A possible origin of this discrepancy is the scaling violation.

\section{Estimating the CKM matrix elements}

We attempt to estimate the CKM matrix elements from our data of $D$ and $D_{s}$ meson masses and decay constants combined with experimental values of leptonic decay widths. Using the CLEO value of $\Gamma\left(D_{s} \rightarrow l v\right)[3]$, we find

$$
\begin{aligned}
\left|V_{c s}\right|(\text { lattice }) & =0.98(2)(3)+O\left(g^{2} a\right), \\
\left|V_{c s}\right|(P D G) & =1.04(6)[1]
\end{aligned}
$$


The first error is statistical, and the second is experimental. For comparison, the PDG value is listed. Our preliminary result is consistent with the PDG value. We note that our estimate of $\left|V_{c s}\right|$ still has a discretization error of $O\left(g^{2} a\right)$ due to the use of perturbative renormalization factor. We must take the continuum extrapolation to remove this source of uncertainty.

We can estimate $\left|V_{c d}\right|$ from the $D$ meson mass and decay constant and CLEO value of $\Gamma(D \rightarrow$ $l v)$ [13]:

$$
\begin{aligned}
\left|V_{c d}\right|(\text { lattice }) & =0.207(2)(9)+O\left(g^{2} a\right), \\
\left|V_{c d}\right|(P D G) & =0.230(11)[1]
\end{aligned}
$$

Our estimate is smaller than the PDG value by about $10 \%$. For completeness we also record the ratio $\left|V_{c s}\right| /\left|V_{c d}\right|$ for which $O\left(g^{2} a\right)$ errors drop out, and is replaced with scaling violation of $O\left(a^{2}\right)$ :

$$
\begin{aligned}
& \frac{\left|V_{c s}\right|}{\left|V_{c d}\right|}(\text { lattice })=4.72(13)(26)+O\left(a^{2}\right), \\
& \frac{\left|V_{c s}\right|}{\left|V_{c d}\right|}(P D G)=4.52(34)[1] .
\end{aligned}
$$

\section{Conclusion}

We measured charm- $u d$ and charm-strange meson masses and decay constants with the relativistic heavy quark action on the $2+1$ dynamical flavor PACS-CS configurations at $a^{-1}=2.2 \mathrm{GeV}$. Since our sea quark masses are close to the physical point, sea quark mass corrections in our results are small and only short extrapolations to the physical point are needed.

We found our results are consistent with the experimental values in two standard deviations. We do not observe discrepancy from experiments in $f_{D_{s}}$.

Combining our data of masses and decay constants with experimental values of leptonic decay widths, CKM matrix elements are evaluated. Our estimates still have discretization errors, which is expected to be $1 \%$ by the order counting. Continuum extrapolation is needed to achieve a few percent accuracy.

\section{Acknowledgments}

Numerical calculations for the present work have been carried out on the PACS-CS computer under the "Interdisciplinary Computational Science Program" of Center for Computational Sciences, University of Tsukuba. This work is supported in part by Grants-in-Aid for Scientific Research from the Ministry of Education, Culture, Sports, Science and Technology (Nos. 16740147, 17340066, 18104005, 18540250, 18740130, 19740134, 20105002, 20340047, 20540248, 20740123, 20740139 ).

\section{References}

[1] Particle Data Group, C. Amsler et al, Phys. Lett. B 667 (2008) 1.

[2] HPQCD and UKQCD Collaboration, E. Follana et al., Phys. Rev. Lett. 100 (2008) 062002. 
[3] CLEO Collaboration., J.P. Alexander et al., Phys. Rev. D 79 (2009) 052001.

[4] C. Aubin et al, Phys. Rev. Lett. 95 (2005) 122002.

[5] Fermilab Lattice and MILC Collaboration, A. Bazavov et al, PoS (LATTICE 2009) 249.

[6] PACS-CS Collaboration, S. Aoki et al., Phys. Rev. D 79 (2009) 034503;

PACS-CS Collaboration, Y. Kuramashi et al., PoS (LATTICE 2009 ) 110.

[7] CP-PACS and JLQCD Collaborations, S. Aoki et al., Phys. Rev. D73, 034501 (2006).

[8] S. Aoki, Y. Kuramashi and S. Tominaga, Prog. Theor. Phys. 109 (2003) 383.

[9] S. Aoki, Y. Kayaba and Y. Kuramashi, Nucl. Phys. B 697 (2004) 271.

[10] PACS-CS Collaboration, Y. Namekawa et al., PoS (LATTICE 2008 ) 121.

[11] S. Aoki, Y. Kayaba and Y. Kuramashi, Nucl. Phys. B 689 (2004) 127.

[12] CP-PACS/JLQCD and ALPHA Collaboration, T. Kaneko et al., JHEP 0704 (2007) 092.

[13] CLEO Collab., B.I.Eisenstein et al., Phys. Rev. D 78 (2008) 052003. 